\title{
An electrical characterisation system for the real-time acquisition of multiple independent sensing parameters from organic thin film transistors
}

\author{
A. Dragoneas, L. Hague, and M. Grell \\ Physics and Astronomy, The University of Sheffield, Hicks Building, Hounsfield Road, S3 7RH, Sheffield, UK \\ Correspondence to: A. Dragoneas (a.dragoneas@mpic.de)
}

Received: 16 November 2014 - Revised: 12 April 2015 - Accepted: 17 April 2015 - Published: 13 May 2015

\begin{abstract}
The presence of multiple independent sensing parameters in a single device is the key conceptual advantage of sensor devices based on an organic thin film transistor (OTFT) over simple organic chemiresistors. Practically, however, these multiple parameters must first be extracted from the electrical characteristics of the OTFTs and, thus, they are not immediately apparent. To exploit the advantage of OTFT sensors, we require a measurement technology to extract these parameters in real time. Here, we introduce an efficient, cost-effective system that is a faster and more compact alternative to the expensive and cumbersome laboratory-based instruments currently available. The characterisation system presented here records the electric behaviour of OTFTs in the form of its "saturated transfer characteristics" multiple times per second for virtually unlimited periods of time, with the option to multiplex up to 20 devices in parallel. By applying a bespoke algorithm to the measured transfer characteristics, the system then extracts, in real time, several underlying transistor parameters (on- and off-current, threshold voltage, and charge carrier mobility). Tests were conducted on the example of a poly(thieno[3,2-b]thiophene) (PBTTT) OTFT exposed to ethanol vapour. The system extracts the underlying OTFT parameters with very low noise without introducing apparent correlations between independent parameters as an artefact.
\end{abstract}

\section{Introduction}

Organic semiconductors (OSCs) are generally more susceptible to environmental factors than traditional (inorganic) semiconductors, so their electronic properties, and consequently the device performance, often strongly decay under ambient atmosphere. This may be due to oxygen, humidity, or pollutants in the air. While such sensitivity is a disadvantage in the context of many applications, it may be an asset for sensor technology. A prominent example is the poly(triaryl amine) (PTAA) family of compounds, which proved unsuitable as realistic photoconductors due to their strong vulnerability to the common air pollutant $\mathrm{NO}_{2}$, yet qualified them for use in $\mathrm{NO}_{2}$ sensors (Das et al., 2007).

A further attraction of OSCs for sensor technology is that their response can be read electrically, which allows for straightforward transduction. A number of "chemiresistors" have been demonstrated, wherein the interaction between an analyte and an OSC is converted to a change in electrical resistance. This may be a drop from a high resistance when OSCs are exposed to dopants, e.g. poly(aniline) (PAni) to acidic vapours (Huang et al., 2003), or polythiophene exposed to humidity (Dragoneas et al., 2013). Alternatively, there may be an increase in resistance in previously doped organic semiconductors ("synthetic metals") exposed to analyte odour that acts as de-doping agents, e.g. doped PAni under amines (Sotzing et al., 2000).

To improve on the simple chemiresistor concept, sensitive OSCs have also been used in organic thin film transistors (OTFTs). In an OTFT, conductivity between two terminals of the device, the "source" (S) and "drain" (D), is enhanced by a sufficiently high voltage of the appropriate polarity applied to a third, insulated terminal, the "gate" (G) (Horowitz, 2000). Under a high gate voltage $\left(V_{\mathrm{G}}\right)$, the induced "saturated drain current" $\left(I_{\mathrm{D} \text {,sat }}\right)$, hereafter referred to as the "on-current" $\left(I_{\mathrm{ON}}\right)$, can be orders of magnitude higher 
than the current of a non-gated chemiresistor, which typically is similar to the OTFTs "off-current", when no $V_{\mathrm{G}}$ is applied. This brings advantages in terms of signal-to-noise ratio (SNR) and thus the limit of detection (LoD); a large number of OTFT sensors have already been reported in the literature (e.g. Crone et al., 2001; Das et al., 2007; Guo et al., 2006; Hague et al., 2011b; Street et al., 2007; Torsi et al., 2008, 2009).

There is also a conceptual advantage of OTFT sensors over chemiresistors: the resistance of a chemiresistor is a single parameter, given simply by the product of charge carrier density and mobility, which are themselves correlated (Hague et al., 2011b). The characteristics of an OTFT (e.g., the saturated transfer characteristics, $\left.I_{\mathrm{D} \text {, sat }}\left(V_{\mathrm{G}}\right)\right)$ may reveal multiple uncorrelated parameters, i.e. carrier mobility $(\mu)$, threshold voltage $\left(V_{\text {th }}\right)$ and on/off ratio; each of these parameters may respond independently and differently to different analytes. OTFT sensors can therefore provide richer information than chemiresistors for the identification of different analytes based upon their effects on several parameters of the same sensor device. Bayn et al. (2013), Wang and Haick (2013a, b), Ermanok et al. (2013), and Wang et al. (2014) have recently shown impressive gas sensing performance of organic and organic/inorganic hybrid TFTs under multiparametric characterisation.

However, to take full advantage of the multiparametric nature of OTFT sensors, we first need to delineate these parameters from measured OTFT characteristics. While the extraction of mobility, threshold, and on/off ratio from OTFT saturated transfer characteristics is well established in principle (Horowitz, 2000), it is a substantial technological challenge to implement this in an automated and portable realtime OTFT measurement system. Bayn et al. (2013), Wang and Haick (2013a, b), Ermanok et al. (2013), and Wang et al. (2014) relied on conventional, laboratory-based semiconductor parameter analysers, which limit the scope for practical applications. Alternative real-time separation between mobility and threshold in sensor OTFTs has been attempted before (Das et al., 2009). However, when mobility and threshold data were fed separately into a genetic algorithm for analyte recognition (Wedge et al., 2009), they proved to be no more effective than using "on-current" $I_{\mathrm{ON}}$ alone. It is not known whether this was due to the noisy nature of extracted parameters, or because they became correlated as an artefact of the system's algorithms.

The advanced measurement solution we present here meets the requirements of thin film transistor sensors. The system is capable of acquiring OTFT saturated transfer $\left(I_{\mathrm{D}, \mathrm{sat}}\left(V_{\mathrm{G}}\right)\right)$ characteristics; by the application of a bespoke numerical data evaluation scheme, it can then extract charge carrier mobility and threshold voltage as uncorrelated parameters with very low noise in real time. To test this instrument, we characterised an OTFT with polymer OSC "PBTTT" (poly(2,5-bis(3-hexadecylthiophen-2yl)thieno(3,2-b)thiophene) under exposure to ethanol (EtOH) odour. This is not meant to lead to a practical sensor device - EtOH odour sensors are well established (fuel-cell based "breathalysers"; Leonard, 2012) - but to provide a realistic yet generic proof-of-concept. However, given the strong scientific interest that PBTTT has attracted since its introduction in 2006 (Mcculloch et al., 2006), studying its behaviour under EtOH has scientific merit in itself: it is known that exposure to EtOH may lead to morphological changes in other thiophene polymers (Jiang-Feng et al., 2011; Nam et al., 2010), but this has not yet been investigated for PBTTT.

\section{Methods}

\subsection{OTFT preparation}

OTFTs were built on heavily n-doped Si substrates with a thermally grown $300 \mathrm{~nm}$ thick oxide. The substrates were cleaned with an alkaline solution and isopropyl alcohol before a UV-ozone treatment was applied. For the sake of reproducibility and simplicity, as well as the exclusion of any additional interactions, no surface treatment was applied to the gate oxide. We used the OSC PBTTT-C16, supplied by Ossila Ltd (product number M141), dissolved in 1,2-dichlorobenzene $\left(7.5 \mathrm{mg} \mathrm{mL}^{-1}\right)$ at $100^{\circ} \mathrm{C}$. PBTTT is more stable under ambient conditions than the common organic semiconductor poly(3-hexyl thiophene) (P3HT), which has well-documented problems with oxygen and humidity (Hoshino et al., 2004). The solution was spincast onto the substrates in a spin coater with a nitrogen purge, at $1500 \mathrm{rpm}$ for $60 \mathrm{~s}$; the substrates were then annealed at $95^{\circ} \mathrm{C}$ for $45 \mathrm{~min}$ in dynamic vacuum. Gold was thermally evaporated through shadow masks in a high vacuum of $5 \times 10^{-7}$ Torr at a rate of $0.03 \mathrm{~nm} \mathrm{~s}^{-1}$. The shadow masks patterned 20 pairs of sourcedrain contacts on each substrate. The channel dimensions of the devices were $5 \mu \mathrm{m}$ (length) by $1000 \mu \mathrm{m}$ (width). The solution preparation and deposition, substrate annealing, gold deposition, as well as all device measurements, were conducted under either dimmed yellow light or in the dark.

\subsection{Odour exposure}

Gas flow and mixing ratios were regulated by two voltagecontrolled mass-flow controllers (Tylan FC-260) with a fullscale throughput of $500 \mathrm{sccm}$ (standard cubic centimetres per minute), which were addressed by a computer using a bespoke LabVIEW application and a NI-USB 6008 device. A solenoid valve was placed before each controller to block the small but undesirable gas flow at the minimum setting of their scales. One mass flow controller regulated the flow of $\mathrm{N}_{2}$ through a "bubbler" bottle to generate saturated analyte odour; the other regulated the flow of pure $\mathrm{N}_{2}$ to dilute the saturated odour when both lines joined at a mixing point. In this way, vapour atmospheres in the range (1 to 0.01 ) $p / p_{\text {sat }}$ could be generated, where $p_{\text {sat }}$ stands for the saturated vapour pressure. $p_{\text {sat }}$ itself depends on the particular analyte (here, ethanol), and the analyte temperature, which can be 

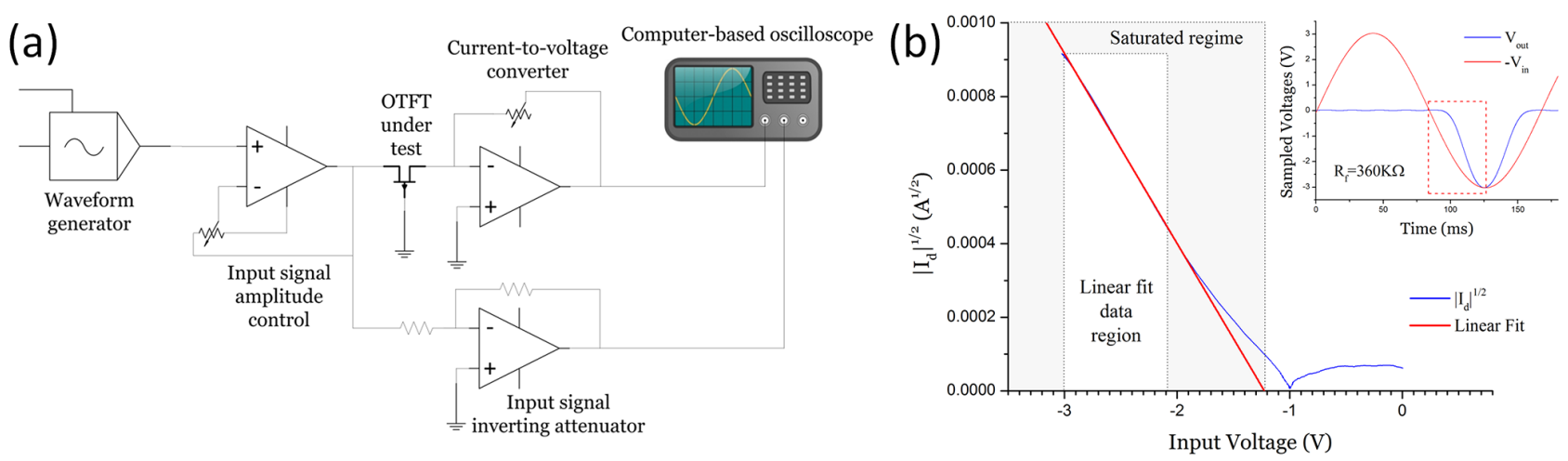

Figure 1. (a) A simplified schematic of the circuit for real-time measurements. (b) Inset: plot of $-V_{\text {in }}$ (red sine) and $V_{\text {out }}$ (blue) versus time, showing "on" and "off" half-cycles. Main plot: saturated transfer characteristic (blue) in the form square root of modulus of drain current versus applied voltage $\left(-V_{\text {in }}\right)$. Same data as in the inset, but time is eliminated. Red straight line: linear fit to high drive voltage data for evaluation of mobility and threshold.

controlled by immersion of the bubbler bottle into a thermostatically controlled water bath. All pipework and the exposure chamber were thoroughly flushed with nitrogen before each test. Transistor devices were placed inside the Teflonlined exposure chamber and contacted by an array of springloaded Au contact pins, which are shown in Fig. 2b.

\subsection{Real-time electrical characterisation}

We designed and built a bespoke, fully automated, computercontrolled, real-time, multiparametric electrical characterisation system for OTFTs. Its operating principle is based upon our previous report on a OTFT characterisation system employing an operational amplifier (op-amp) (Hague et al., 2011a). The portable system was interfaced to a PC via a Pico Technology Picoscope 2204 device, which acts as both a two-channel digital oscilloscope and a waveform generator. The circuit is mainly built around three op-amps with different specifications. A high-voltage op-amp (Texas Instruments OPA445AP) is used to amplify a zero-offset, lowfrequency $(f=6 \mathrm{~Hz})$ sine drive provided by the Picoscope waveform generator, to extend the limited voltage amplitude of its output. A zero-offset sine waveform is chosen to minimise gate dielectric stress (Zschieschang et al., 2009) thanks to its symmetry, and to avoid higher harmonics. The amplified sinusoidal drive signal, $V_{\text {in }}\left(=V_{\text {MAX }} \sin (\omega(t))\right)$, is fed into the source of the OTFT under test, while the gate is connected to the electrical ground and the drain is connected to virtual ground (see below). Therefore, this drive configuration is equivalent to connecting the source to ground and applying $-V_{\text {in }}$ to both the drain and gate. A hole (or electron) transporting OTFT will turn "on" for a sufficiently large positive (or negative) $V_{\text {in }}$, and deliver a saturated drain current, $I_{\mathrm{D} \text {,sat }}\left(V_{\mathrm{G}}=V_{\mathrm{D}}=-V_{\text {in }}\right) . V_{\text {in }}$ is "sufficiently" large when it has the same polarity as, and its modulus exceeds the modulus of, threshold, $V_{\text {th }}$. The resulting saturated drain current is fed into the inverting input of a high-input impedance op- amp (Analog Devices AD549JH), which is configured as a current / voltage $(I / V)$ converter by feeding back the output voltage $\left(V_{\text {out }}\right)$ to the inverting input via a high-precision feedback resistor, $R_{\mathrm{f}}$ (here, $R_{\mathrm{f}}=1 \mathrm{M} \Omega$ ). The non-inverting input of the same op-amp is grounded, thus establishing virtual ground at the inverting input. $V_{\text {out }}$ is related to the $I_{\mathrm{D} \text {, sat }}$ of the OTFT via the equation $V_{\text {out }}=-R_{\mathrm{f}} I_{\mathrm{D} \text {, sat }}$. The third op-amp (Linear Technology LT1677) is configured as a lownoise attenuator and inverter of the input signal $\left(V_{\text {in }}\right)$, which provides a reference voltage $\left(V_{\text {ref }}=-0.1 V_{\text {in }}\right)$ which can be safely sampled by the oscilloscope and used to trigger the measurement. The output voltage $\left(V_{\text {out }}\right)$, which is proportional to $I_{\mathrm{D} \text {,sat }}$, and the attenuated and inverted input voltage $\left(V_{\text {ref }}\right)$, are connected to the two oscilloscope channels of the Picoscope, which carry out the analogue-to-digital conversion. Together, $V_{\text {out }}(t)$ and $10 V_{\text {ref }}(t)=-V_{\text {in }}(t)$ represent the saturated transfer characteristic of the OTFT, parametric in time. Figure 1a schematically illustrates the system.

A National Instruments LabVIEW application has been developed to fully automate the characterisation process. This application collects the sampled data from the oscilloscope and plots the input and output voltages versus time as shown in the inset to Fig. 1b. Low-pass digital filters can also be applied. As highlighted by the dashed red rectangle in the inset to Fig. 1b, for all calculations presented here, the third quadrant of the sinusoidal signal period was used; i.e. the rising flank of the input voltage $\left(V_{\text {in }}\right)$. Drain current at every sampling point in time $(t)$ can be calculated according to

$I_{\mathrm{d}}\left(V_{\text {in }}(t)\right)=-\frac{V_{\text {out }}(t)}{R_{\mathrm{f}}}$.

Then, the root of drain current is plotted against $V_{\text {in }}$ directly, eliminating time. This agrees with the common $I_{\mathrm{D}, \mathrm{sat}}{ }^{1 / 2}$ versus $V_{\mathrm{G}}$ plot of saturated OTFT transfer characteristics, which is routinely used to evaluate charge carrier mobility $(\mu)$ and threshold voltage $\left(V_{\text {th }}\right)$ (Locklin and Bao, 2006) from slope and intercept. Figure $1 b$ shows an exam- 
(a)

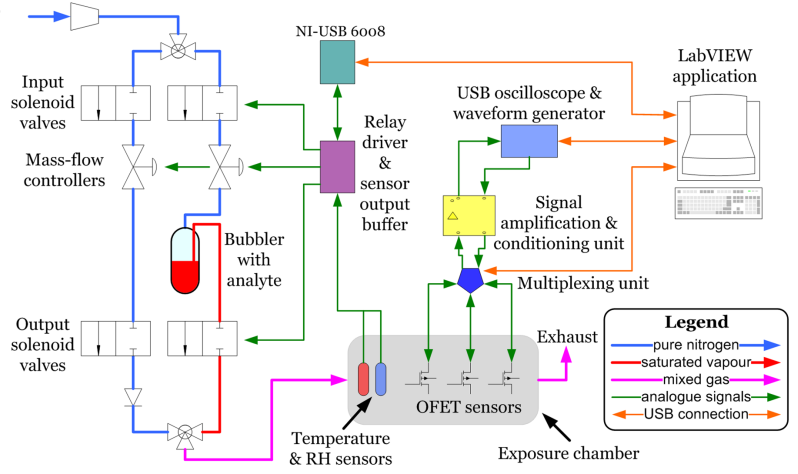

(b)

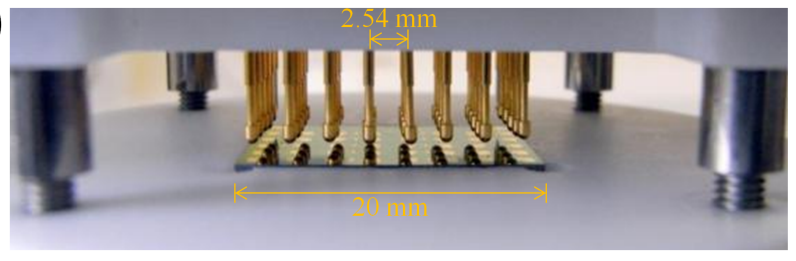

Figure 2. (a) Schematic representation of the computer-controlled pneumatic analyte odour delivery system, and multiplexed electrical OTFT drive and measurement system. (b) Array of 40 springloaded Au contact pins, matching the $S / D$ contacts for the 20 OTFTs on the OTFT substrate below, shortly before making contact inside the exposure chamber.

ple: at high $V_{\text {in }}$ we indeed find a good fit to a straight line. It is worth noting that here, drain voltage ( $\left.V_{\mathrm{DS}}\right)$ is swept simultaneously with the gate voltage $\left(V_{\mathrm{GS}}\right)$ rather than being kept constant, as in conventional transfer sweeps. This different convention results in the uncommon subthreshold behaviour shown in Fig. 1b. However, for high $V_{\text {in }}$, the curve coincides with the conventional saturated transfer characteristics. We therefore apply a simple linear regression fit in the high $V_{\text {in }}$ regime of the $\left(I_{\mathrm{D}, \text { sat }}\right)^{1 / 2}$ versus $V_{\text {in }}$ plot; in this study, the data range for the fit was in the range $V_{\text {in }}=-(0.707$ to 1$)$ $V_{\text {MAX }}$. Threshold voltage is extracted as the intercept of the extrapolated straight line with the $V_{\text {in }}$ axis. We note that this procedure is robust against a transition from "normally off" to "normally on" behaviour, which we did in fact sometimes observe under exposure (see Sect. 3.2). Carrier mobility $(\mu)$ was evaluated from the slope $(s)$ of the fit line, according to

$\mu=\frac{2 L}{W C_{\mathrm{i}}} s^{2}$,

wherein $W$ and $L$ stand for the width and length of the transistor's channel, respectively, and $C_{\mathrm{i}}$ is the gate insulator's specific capacitance, here $C_{\mathrm{i}}=10 \mathrm{nF} \mathrm{cm}^{-2}$. Equation (2) derives from the common equation for saturated drain current in an OTFT (Horowitz, 2000),

$I_{\mathrm{d}, \mathrm{sat}}=\frac{W}{2 L} \mu C_{\mathrm{i}}\left(V_{\mathrm{G}}-V_{\mathrm{th}}\right)^{2}$.

$I_{\mathrm{ON}}$ and $I_{\mathrm{OFF}}$ are simply defined as the currents recorded at $V_{\text {in }}= \pm V_{\text {MAX }}$, i.e. maximum/minimum $I_{\mathrm{d}}$. At a scanning speed $f=6 \mathrm{~Hz}$, it is possible to get six sets of data ( $I_{\mathrm{ON}}$, $\left.I_{\mathrm{OFF}}, \mu, V_{\mathrm{th}}\right)$ per second, which are subsequently filtered by a median filter. The system is capable of supporting multiple sensors to allow for simultaneous comparative measurements; this attribute makes the system appropriate for applications such as "electronic noses". This is achieved by the use of one of two bespoke computer-controlled multiplexers; one of them is a relay-based circuit which is controlled by the NI-USB 6008 unit, whereas the other is a stand-alone microcontroller-based system, utilising solid-state analogue switches, which communicates with the computer over a USB connection. The maximum supported drive voltages are \pm 45 and $\pm 8 \mathrm{~V}$ for the relay-based and solid-state units, respectively. A schematic representation of the described odour exposure and electrical characterisation scheme is shown in Fig. 2a.

OTFT threshold $\left(V_{\mathrm{th}}\right)$ and mobility $(\mu)$ are the subject of theoretical treatments, e.g. by Horowitz et al. for $V_{\text {th }}$ (Horowitz, 2000; Horowitz et al., 1998), and Bässler for $\mu$ (Bässler, 1993). Extracting these from measured saturated transfer characteristics, therefore, in principle provides an opportunity to relate observed OTFT response under odour exposure to the physical interactions between analyte and OSC. From the band model for carrier injection into OTFTs, Horowitz et al. (1998) give Eq. (4) for $V_{\text {th }}$ :

$V_{\mathrm{th}}= \pm \frac{q n_{0} d_{\mathrm{s}}}{C_{\mathrm{i}}}+V_{\mathrm{FB}}$

wherein \pm applies to hole/electron transporting semiconductors, $q$ is the elementary charge, $n_{0}$ the doping level in the semiconductor, $d_{\mathrm{s}}$ the thickness of the semiconducting film, and $V_{\mathrm{FB}}$ the flat-band voltage. $V_{\mathrm{FB}}$ contains a number of contributions, including the metal work function, $\Phi_{\mathrm{m}}$.

Equation (4) suggests how odour can influence $V_{\text {th }}$ : either by doping (via $n_{0}$ ) or by adsorption of polar molecules on an interface, which changes $\Phi_{\mathrm{m}}$ and, hence, $V_{\mathrm{FB}}$. Bässler's model of organic mobility is more intricate, and involves semi-empirical "disorder parameters", which may also be impacted by organic odour. However, there may be further interactions not explicitly covered by these models (e.g. deep carrier traps).

\section{Results and discussion}

\subsection{PBTTT OTFT long-term stability}

Figure 3 shows both the on- and off-currents of a PBTTT OTFT recorded over several days using the characterisation system described above with $V_{\text {MAX }}=20 \mathrm{~V}$, while the exposure chamber was continuously purged with dry nitrogen.

An initial off-current (possibly due to unintentional doping during device fabrication) was minimised over time, dropping from 200 to $65 \mathrm{nA}$ over 3 days. The on-current also drops over time (possibly because charge carrier mobility also decreases with decreasing doping levels; Hague 

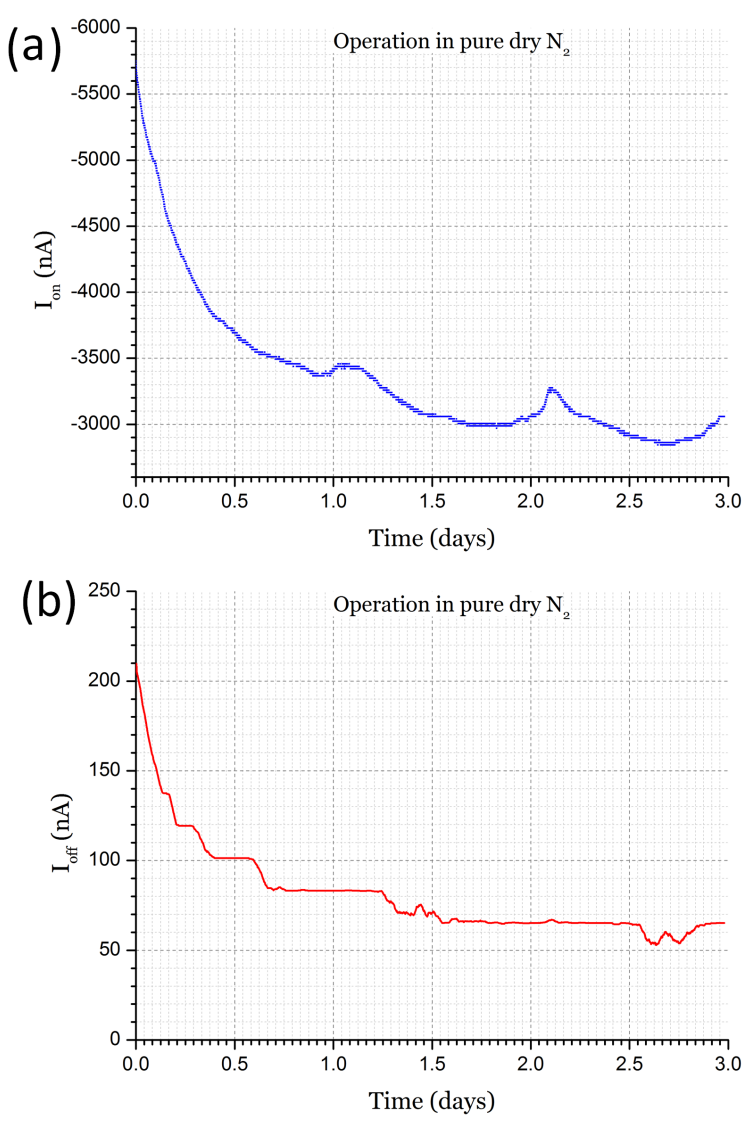

Figure 3. (a) Maximum drain current $\left(I_{\mathrm{ON}}\right)$; (b) minimum drain current $\left(I_{\mathrm{OFF}}\right)$ for a PBTTT OTFT under nitrogen purge over the course of 3 days. Data for $V_{\mathrm{DS}}=V_{\mathrm{GS}}=-20 \mathrm{~V}\left(I_{\mathrm{ON}}\right)$, $V_{\mathrm{DS}}=V_{\mathrm{GS}}=+20 \mathrm{~V}\left(I_{\mathrm{OFF}}\right)$.

et al., 2011b), with some undulations on a $\sim 24 \mathrm{~h}$ timescale, which could be attributed to the effects of daily room temperature fluctuations on charge carrier mobility. Later experiments were taken on a transistor pre-conditioned by a 3-day dry $\mathrm{N}_{2}$ purge, after current drift with time had largely ceased. The initial study presented in Fig. 3 confirms that PBTTT OTFTs display sufficient stability against spontaneous drift on the timescale $(\sim 1 \mathrm{~h})$ of the EtOH exposure/recovery tests reported here.

\subsection{Testing the system with PBTTT OTFTs under exposure to ethanol odour}

To test the multiparametric data acquisition system described above on a practical example, we ran exposure/recovery cycles of PBTTT OTFTs to ethanol (EtOH) odour. In Fig. 4, we present the observed on- and off-current, $I_{\mathrm{ON}}$ and $I_{\mathrm{OFF}}$. We selected EtOH concentrations of 5, 15 and $45 \%$ of $p_{\text {sat }}$ at an ambient temperature of $\sim 20^{\circ} \mathrm{C}$, corresponding to 2750,8250 and $24750 \mathrm{ppm}$, beginning with the lowest concentration. Both $I_{\mathrm{ON}}$ and $I_{\mathrm{OFF}}$ display a distinct response to EtOH exposure. For the two lowest concentra-

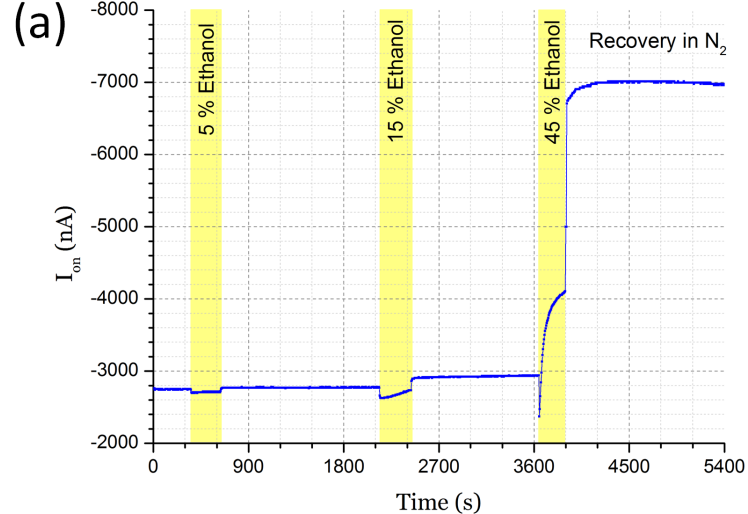

(b)

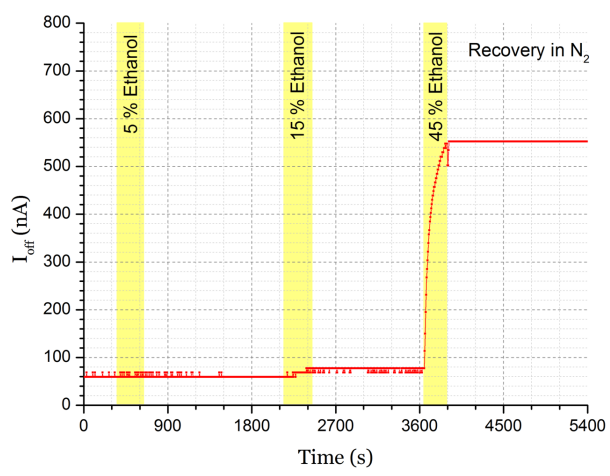

Figure 4. (a) $I_{\mathrm{ON}}\left(V_{\mathrm{DS}}=V_{\mathrm{GS}}=-20 \mathrm{~V}\right)$ under EtOH exposure/ recovery cycles. All white regions depict purge under $500 \mathrm{sccm}$ pure dry nitrogen. Shaded regions indicate exposure to different concentrations of EtOH vapour. From left to right: $5 \% p_{\text {sat }}$ (equivalent to $2750 \mathrm{ppm}), 15 \% p_{\text {sat }}(8250 \mathrm{ppm})$ and $45 \% p_{\text {sat }}(24750 \mathrm{ppm})$. (b) Similar for $I_{\mathrm{OFF}}\left(V_{\mathrm{DS}}=V_{\mathrm{GS}}=+20 \mathrm{~V}\right)$.

tion levels ( 5 and $15 \% p_{\text {sat }}$ ), the $I_{\mathrm{OFF}}$ showed a small rise, whereas $I_{\mathrm{ON}}$ instantly dropped but slowly increased again over time during $15 \% p_{\text {sat }}$ exposure. Immediately after exposure, an instant recovery to a level slightly above the initial $I_{\mathrm{ON}}$ was recorded. The change in drain current under $5 \%$ EtOH can be expressed as a sensitivity $S=16 \mathrm{pAppm}^{-1}$. We note that current versus time traces prior to exposure display very low noise: the scatter of measured data is limited to a single digit in the "quantisation" of analogue-todigital (ADC) conversion, which at $R_{\mathrm{f}}=1 \mathrm{M} \Omega$ corresponds to approximately $16 \mathrm{nA}$. From current noise, we calculate the limit of detection (LoD) as LoD $\sim 16 \mathrm{nA} /\left(16 \mathrm{pA} \mathrm{ppm}^{-1}\right)$ $=1000 \mathrm{ppm}=0.1 \% \mathrm{vol} / \mathrm{vol}$, compared to a lower explosive limit (LEL) of $3.5 \% \mathrm{vol} / \mathrm{vol}$ for EtOH (http://www.distill. com/materialsafety/msds-eu.html).

The most intriguing behaviour was observed under $45 \%$ $p_{\text {sat }} I_{\mathrm{OFF}}$ rose steadily over time without showing any recovery after the period of exposure; the behaviour of $I_{\mathrm{ON}}$ seemed erratic, with an instant drop at the beginning of exposure, followed by a steady increase with time during exposure and a 

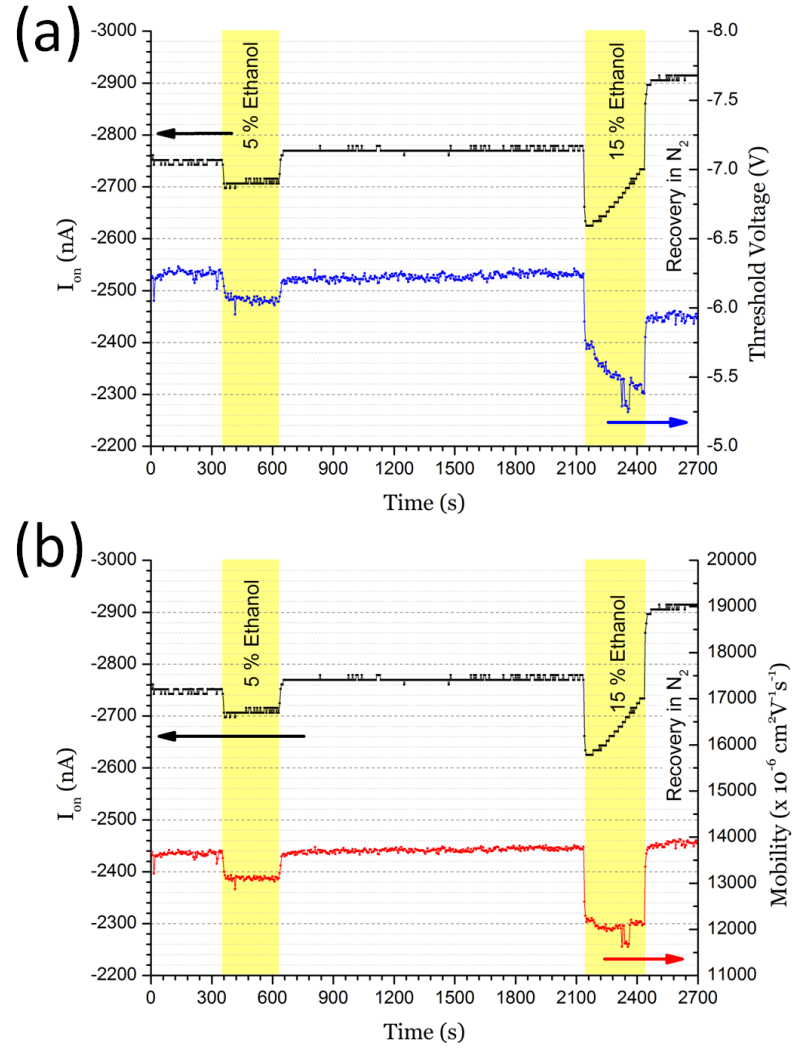

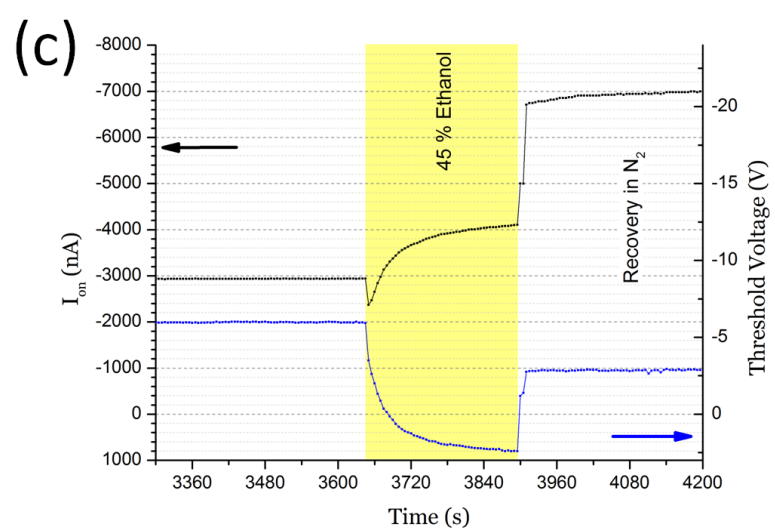

(d)

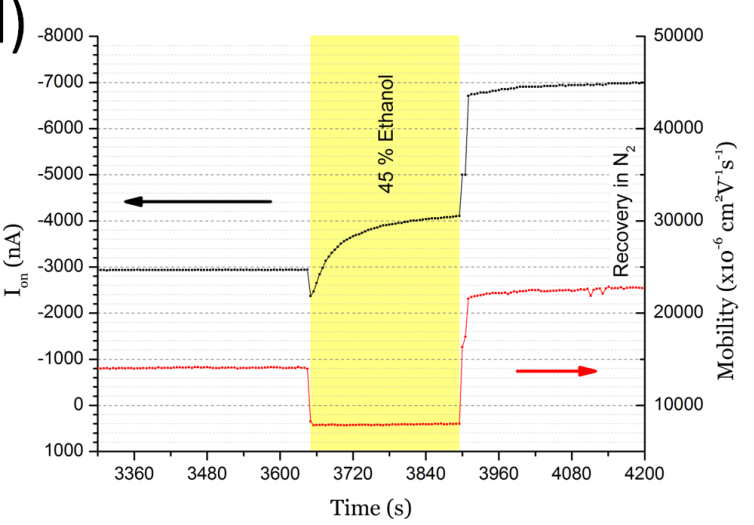

Figure 5. Exposure of PBTTT OTFTs to EtOH. (a) $I_{\mathrm{ON}}$ (top, black curve) and $V_{\text {th }}$ (bottom, blue curve) versus time under $5 / 15 \% p_{\text {sat }}$ EtOH. (b) $I_{\mathrm{ON}}$ (top, black curve) and $\mu$ (bottom, red curve) versus time under $5 / 15 \% p_{\text {sat }}$ EtOH. White regions depict dry nitrogen purge under $500 \mathrm{sccm}$. Shaded regions indicate exposure to different concentrations of ethanol vapour. Left region: $5 \% p_{\text {sat }}(2750 \mathrm{ppm}) \mathrm{EtOH}$; right region: $15 \% p_{\text {sat }}(8250 \mathrm{ppm}) \mathrm{EtOH}$. (c) Similar to (a) but for exposure to $45 \% p_{\text {sat }}(24750 \mathrm{ppm})$ EtOH. (d) Similar to (b) but under $45 \% p_{\text {sat }}(24750 \mathrm{ppm}) \mathrm{EtOH}$.

further step-like increase at the end of exposure to more than twice the prior level, with no subsequent recovery.

The $I_{\mathrm{ON}} / I_{\mathrm{OFF}}$ data shown in Fig. 4 could have also been recorded with a less sophisticated data acquisition/evaluation system; however, the "zigzag" behaviour of $I_{\mathrm{ON}}$ under $45 \%$ $p_{\text {sat }}$ is particularly difficult to rationalise without further information. For the intended purpose of demonstrating the technical quality, and scientific merit, of this multiparametric characterisation system, a PBTTT OTFT under EtOH is thus a fortunate choice: only a sophisticated system, like the one developed here, can deliver separate mobility and threshold data with real-time resolution, which will be needed to gain a deeper understanding of such rapid and complex sensor responses.

Figure 5 shows the individual evolution of threshold voltage $\left(V_{\text {th }}\right)$ and carrier mobility $(\mu)$ under exposure to 5,15 , and $45 \% p_{\text {sat }} \mathrm{EtOH}$, as evaluated in real time by the algorithm described above. Note the good quality (low noise) of the $V_{\mathrm{th}} / \mu$ data. For reference, $I_{\mathrm{ON}}$ is also shown.

It can be seen in Fig. 5 that both $V_{\text {th }}$ and $\mu$ are affected by $\mathrm{EtOH}$, showing stronger responses for higher EtOH concentrations. In all cases, mobility immediately drops to a lower level and then remains constant throughout each exposure period; the modulus of $V_{\text {th }}$ drops instantly and then gradually reduces further throughout the exposure period, in an approximately exponential curve. Under $45 \% p_{\text {sat }}, V_{\text {th }}$ even turns slightly positive, indicating a transition from a "normally off" (enhancement-mode) to a "normally on" (depletion-mode) type of OTFT. Significantly, under EtOH exposure, one parameter $\left(V_{\text {th }}\right)$ is shown to slowly evolve in time, while another $(\mu)$ remains constant after a rapid, initial change. This underscores the independence of the extracted parameters: this data analysis system does not introduce correlations between parameters as an artefact, when they are physically independent. Otherwise, real drift in one parameter would necessarily lead to apparent drift in the other.

According to Eq. (3), the trends in $V_{\text {th }}$ and $\mu$ have opposite effects on $I_{\mathrm{ON}}$ : a drop in (the modulus of) $V_{\text {th }}$ leads to an increase, whereas a drop in $\mu$ leads to a decrease. The initial drop/later gradual rise of $I_{\mathrm{ON}}$ under EtOH can thus be explained by the different dynamics of opposing trends.

At the end of analyte exposure, both threshold voltage and mobility responded quickly. For exposure to low $\mathrm{EtOH}$ odour concentration $\left(5 \% p_{\text {sat }}\right)$, recovery is almost complete, i.e. 
sensor response is almost fully reversible. However, under exposure to higher EtOH concentrations, PBTTT OTFTs undergo irreversible changes. After exposure to $45 \% p_{\text {sat }}, V_{\text {th }}$ returned to a "normally off" state, yet with a lower modulus than prior to exposure; meanwhile, mobility showed a reduction under exposure, which was followed by a recovery to a level higher than its pre-exposure value. Immediately after each exposure period, both reduced threshold voltage and increased mobility contributed to a higher overall $I_{\mathrm{ON}}$, as compared to its value prior to exposure ( $I_{\mathrm{ON}}$ more than doubles after $45 \% p_{\text {sat }}$ EtOH exposure). $I_{\mathrm{ON}}$ does not return to its previous value, even when purged under dry nitrogen for more than $2 \mathrm{~h}$ after the last exposure. Exposure to $\mathrm{EtOH}$ thus leads to both reversible, and irreversible, changes in mobility and threshold, which we will discuss separately.

The results for PBTTT OTFTs under low EtOH concentrations are similar to those from previous work on a polythiophene derivative OTFT by Torsi et al. (2004), who reported a quick and reversible reduction of $I_{\mathrm{ON}}$ by $\sim 0.2 \%$ under $700 \mathrm{ppm}=1.3 \% p_{\text {sat }} \mathrm{EtOH}$, albeit without delineating into mobility and threshold voltage changes. This is comparable to the observation, given here, of a largely reversible $\sim 1.8 \%$ reduction of $I_{\mathrm{ON}}$ under $5 \% p_{\text {sat }}(2750 \mathrm{ppm})$ EtOH. Such reversible sensing may result from a manifold of possible interactions between analytes and the different components of an OTFT, e.g. doping or de-doping the OSC (Dragoneas et al., 2013; Sotzing et al., 2000), swelling (AlQahtani et al., 2012), assisted tunnelling at OSC grain boundaries (Hague et al., 2011a), changing work function by interfacial adsorption of polar molecules, and introduction of trap sites at the insulator surface. However, none of these lead to permanent morphological changes in the PBTTT film, and as EtOH escapes under nitrogen purge, electrical characteristics largely return to their values prior to exposure.

In contrast, exposure of thiophene OTFTs to more concentrated EtOH odour up to $45 \% p_{\text {sat }}$ leads to irreversible changes that persist under subsequent purge. Such changes must therefore be assigned to morphological changes under $\mathrm{EtOH}$, a process which is referred to as "vapour annealing". Exposure of PBTTT devices under highly concentrated EtOH odour has not previously been reported in the literature. However, it is known that the treatment of the chemically related OSC P3HT with liquid EtOH leads to irreversible morphological changes with improved carrier mobility, either by adding EtOH as a non-solvent to P3HT processing solution prior to casting (Jiang-Feng et al., 2011), or by application of a liquid droplet to a film after casting (Nam et al., 2010). We believe that exposure of PBTTT to concentrated EtOH vapour leads to similar morphological improvements, which partly explain the observed irreversible changes of OTFT parameters. Detailed X-ray diffraction studies of other conjugated polymers also have shown that solvent vapour annealing can significantly and irreversibly increase crystallinity, often more than thermal annealing (e.g. Grell et al., 1999). At this point, it is worth noting that these changes can compromise the sensing capabilities of these transistors and, thus, these devices should not be reused as sensors after exposure to such high analyte concentrations. However, irreversible morphological changes under concentrated odours are specific to a particular OSC-analyte combination, while the focus of this study is the characterisation system that can be applied to all odour-sensing OTFTs.

\section{Conclusions}

"Electronic noses" require the acquisition of manifold information-rich sensor data, and powerful data analysis algorithms to identify and quantify analytes. We here present a comprehensive and reliable system for the acquisition of lownoise, multiparametric, mutually independent OTFT sensor data in real time, which can optionally be used for sensor arrays via multiplexing. The bespoke solution presented here comes with a much lighter footprint and significantly lower cost than commercially available semiconductor parameter analysers; this system enables the user to fully exploit the conceptual advantages of OTFT-based sensors over organic chemiresistors.

Tests of this measurement system were conducted by exposing PBTTT OTFTs to ethanol (EtOH) vapour. $I_{\mathrm{ON}}$ displayed apparently erratic behaviour, particularly under high EtOH concentrations. However, we succeeded in extracting threshold voltage and mobility as independent, uncorrelated parameters with low noise. When inspected separately, mobility and threshold voltage display a more straightforward behaviour, which, taken together, accounted for the observed $I_{\mathrm{ON}}$ behaviour.

While we are confident that morphological changes under concentrated EtOH account in part for the observed behaviour, we cannot provide a full explanation on a molecular level. However, practical "electronic noses" do not rely on detailed interpretations of analyte/sensitiser interactions either, but pragmatically use measured exposure data as a "feedstock" to train advanced pattern recognition algorithms; we refer to the review by Scott et al. (2006). Such an approach is particularly appropriate in the case of OTFT sensors, where the theory of carrier mobility and threshold voltage is complex, and still involves empirical parameters, even in the absence of analytes [5, 21, 22].

The measurement technology solution for the acquisition of multiple independent sensing parameters from organic thin film transistors described here is well suited to deliver a rich data feedstock for such algorithms. 
Acknowledgements. A. Dragoneas would like to acknowledge the European Commission for the provision of an Early Stage Researcher (ESR) Marie Curie fellowship within the FlexSmell ITN of the 7th Framework Programme (FP7). L. Hague would like to thank the Engineering and Physical Sciences Research Council (EPSRC) for the provision of his Doctoral Prize Fellowship.

Edited by: M. Kraft

Reviewed by: four anonymous referees

\section{References}

AlQahtani, H., Alduraibi, M., Richardson, T., and Grell, M.: Manifold sensitivity improvement of swelling-based sensors, Phys. Chem. Chem. Phys., 14, 5558-5560, doi:10.1039/C2cp00003b, 2012.

Bässler, H.: Charge transport in disordered organic photoconductors - a monte-carlo simulation study, Phys. Status Solidi B, 175, 1556, doi:10.1002/pssb.2221750102, 1993.

Bayn, A., Feng, X. L., Mullen, K., and Haick, H.: Field effect transistors based on polycyclic aromatic hydrocarbons for the detection and classification of volatile organic compounds, Acs. Appl. Mater. Inter., 5, 3431-3440, doi:10.1021/Am4005144, 2013.

Crone, B., Dodabalapur, A., Gelperin, A., Torsi, L., Katz, H. E., Lovinger, A. J., and Bao, Z.: Electronic sensing of vapors with organic transistors, Appl. Phys. Lett., 78, 2229-2231, doi:10.1063/1.1360785, 2001.

Das, A., Dost, R., Richardson, T., Grell, M., Morrison, J. J., and Turner, M. L.: A nitrogen dioxide sensor based on an organic transistor constructed from amorphous semiconducting polymers, Adv. Mater., 19, 4018-4023, doi:10.1002/adma.200701504, 2007.

Das, A., Dost, R., Richardson, T. H., Grell, M., Wedge, D. C., Kell, D. B., Morrison, J. J., and Turner, M. L.: Low cost, portable, fast multiparameter data acquisition system for organic transistor odour sensors, Sensor. Actuat. B-Chem., 137, 586-591, doi:10.1016/j.snb.2009.01.006, 2009.

Dragoneas, A., Grell, M., Hampton, M., and Macdonald, J. E.: Morphology-driven sensitivity enhancement in organic nanowire chemiresistors, Sensor Lett., 11, 552-555, doi:10.1166/S1.2013.2917, 2013.

Ermanok, R., Assad, O., Zigelboim, K., Wang, B., and Haick, H.: Discriminative power of chemically sensitive silicon nanowire field effect transistors to volatile organic compounds, Acs. Appl. Mater. Inter., 5, 11172-11183, doi:10.1021/Am403421g, 2013.

Grell, M., Bradley, D. D. C., Ungar, G., Hill, J., and Whitehead, K. S.: Interplay of physical structure and photophysics for a liquid crystalline polyfluorene, Macromolecules, 32, 5810-5817, doi:10.1021/Ma990741o, 1999.

Guo, X., Myers, M., Xiao, S., Lefenfeld, M., Steiner, R., Tulevski, G. S., Tang, J., Baumert, J., Leibfarth, F., Yardley, J. T., Steigerwald, M. L., Kim, P., and Nuckolls, C.: Chemoresponsive monolayer transistors, P. Natl. Acad. Sci. USA, 103, 11452-11456, doi:10.1073/pnas.0601675103, 2006.

Hague, L., Puzzovio, D., Dragoneas, A., and Grell, M.: Simplified real-time organic transistor characterisation schemes for sensing applications, Sci. Adv. Mat., 3, 907-911, doi:10.1166/sam.2011.1216, 2011a.
Hague, L., Puzzovio, D., Richardson, T. H., and Grell, M.: Discovery of a new odour sensing mechanism using an n-type organic transistor, Sensor Lett., 9, 1692-1696, doi:10.1166/S1.2011.1734, 2011b.

Horowitz, G.: Physics of organic field-effect transistors, in: Semiconducting polymers, edited by: Hadziioannou, G. and Malliaras, G. G., 463-514, 2000.

Horowitz, G., Hajlaoui, R., Bouchriha, H., Bourguiga, R., and Hajlaoui, M.: The concept of "threshold voltage" in organic field-effect transistors, Adv Mater, 10, 923927, doi:10.1002/(Sici)1521-4095(199808)10:12<923::AidAdma923>3.0.Co;2-W, 1998.

Hoshino, S., Yoshida, M., Uemura, S., Kodzasa, T., Takada, N., Kamata, T., and Yase, K.: Influence of moisture on device characteristics of polythiophene-based field-effect transistors, J. Appl. Phys., 95, 5088-5093, doi:10.1063/1.1691190, 2004.

Huang, J. X., Virji, S., Weiller, B. H., and Kaner, R. B.: Polyaniline nanofibers: Facile synthesis and chemical sensors, J. Am. Chem. Soc., 125, 314-315, doi:10.1021/Ja028371y, 2003.

Jiang-Feng, Y., Su-Ling, Z., Zheng, X., Jiang-Feng, Y., Fu-Jun, Z., and Xue-Yan, T.: Non-solvent addition induced self-organization for enhancement of performance of poly(3-hexylthiophene) organic field-effect transistors, Acta Phys. Sin., 60, 37201-037201, doi:10.7498/aps.60.037201, 2011.

Leonard, R. J.: Evaluation of the analytical performance of a fuel cell breath alcohol testing instrument: A seven-year comprehensive study, J. Forensic Sci., 57, 1614-1620, doi:10.1111/j.15564029.2012.02146.x, 2012.

Locklin, J. and Bao, Z. N.: Effect of morphology on organic thin film transistor sensors, Anal. Bioanal. Chem., 384, 336-342, doi:10.1007/s00216-005-0137-z, 2006.

Mcculloch, I., Heeney, M., Bailey, C., Genevicius, K., MacDonald, I., Shkunov, M., Sparrowe, D., Tierney, S., Wagner, R., Zhang, W. M., Chabinyc, M. L., Kline, R. J., Mcgehee, M. D., and Toney, M. F.: Liquid-crystalline semiconducting polymers with high charge-carrier mobility, Nat. Mater., 5, 328-333, doi:10.1038/Nmat1612, 2006.

Nam, S., Chung, D. S., Jang, J., Kim, S. H., Yang, C., Kwon, S. K., and Park, C. E.: Effects of poor solvent for solution-processing passivation of organic field effect transistors, J. Electrochem. Soc., 157, H90-H93, doi:10.1149/1.3251337, 2010.

Scott, S. M., James, D., and Ali, Z.: Data analysis for electronic nose systems, Microchim. Acta, 156, 183-207, doi:10.1007/s00604006-0623-9, 2006.

Sotzing, G. A., Phend, J. N., Grubbs, R. H., and Lewis, N. S.: Highly sensitive detection and discrimination of biogenic amines utilizing arrays of polyaniline/carbon black composite vapor detectors, Chem. Mater., 12, 593-595, doi:10.1021/Cm990694e, 2000.

Street, R. A., Chabinyc, M. L., and Endicott, F.: Chemical impurity effects on transport in polymer transistors, Phys. Rev. B, 76, 045208, doi:10.1103/Physrevb.76.045208, 2007.

Torsi, L., Tanese, M. C., Cioffi, N., Gallazzi, M. C., Sabbatini, L., and Zambonin, P. G.: Alkoxy-substituted polyterthiophene thinfilm-transistors as alcohol sensors, Sensor. Actuat. B-Chem., 98, 204-207, doi:10.1016/j.snb.2003.10.007, 2004.

Torsi, L., Farinola, G. M., Marinelli, F., Tanese, M. C., Omar, O. H., Valli, L., Babudri, F., Palmisano, F., Zambonin, P. G., and Naso, 
F.: A sensitivity-enhanced field-effect chiral sensor, Nat. Mater., 7, 412-417, doi:10.1038/Nmat2167, 2008.

Torsi, L., Marinelli, F., Angione, M. D., Dell'Aquila, A., Cioffi, N., De Giglio, E., and Sabbatini, L.: Contact effects in organic thin-film transistor sensors, Org. Electron., 10, 233-239, doi:10.1016/j.orgel.2008.11.009, 2009.

Wang, B. and Haick, H.: Effect of functional groups on the sensing properties of silicon nanowires toward volatile compounds, Acs. Appl. Mater. Inter., 5, 2289-2299, doi:10.1021/Am4004649, 2013a.

Wang, B. and Haick, H.: Effect of chain length on the sensing of volatile organic compounds by means of silicon nanowires, Acs. Appl. Mater. Inter., 5, 5748-5756, doi:10.1021/Am401265z, $2013 b$.
Wang, B., Cancilla, J. C., Torrecilla, J. S., and Haick, H.: Artificial sensing intelligence with silicon nanowires for ultraselective detection in the gas phase, Nano Lett., 14, 933-938, doi:10.1021/N1404335p, 2014.

Wedge, D. C., Das, A., Dost, R., Kettle, J., Madec, M. B., Morrison, J. J., Grell, M., Kell, D. B., Richardson, T. H., Yeates, S., and Turner, M. L.: Real-time vapour sensing using an ofetbased electronic nose and genetic programming, Sensor. Actuat B-Chem., 143, 365-372, doi:10.1016/j.snb.2009.09.030, 2009.

Zschieschang, U., Weitz, R. T., Kern, K., and Klauk, H.: Bias stress effect in low-voltage organic thin-film transistors, Appl. Phys A-Mater., 95, 139-145, doi:10.1007/s00339-008-5019-8, 2009. 